MITSUBISHI ELECTRIC RESEARCH LABORATORIES

http://www.merl.com

\title{
Autonomous Field-deployable Device for the measurement of Phosphate in Natural Water
}

\author{
Conor Slater, John Cleary, Christina McGraw, William Yerazunis, King Tong Lau \\ TR2007-105 September 2007
}

\begin{abstract}
This work describes the ongoing development of an autonomous platform for the measurement of phosphate levels in river water. This device is designed to operate unassisted for one year, taking a measurement every hour and relaying the result to a laptop computer. A first generation prototype has already been developed and successfully field tested. The system contains the sampling, chemical storage, fluid handing, colorimetric data acquisition and waste storage capabilities necessary to perform the phosphate measurement. In addition to this, the device has the embedded control, GSM communications system and power supply to allow independent operation. The entire system is placed inside a compact and rugged enclosure. Further work discussed here builds on the successes of the prototype design to deliver a system capable of one full year of operation. The second generation system has been built from the ground up. Although identical in operation of the prototype its design has a greater emphasis on power efficient components and power management to allow for a longer lifetime. Other improvements include an automated two-point calibration to compensate for drift and a more rugged design to further increase the lifetime of the device.
\end{abstract}

SPIE Advanced Enfironmental, Chemical and Biological Sensing Technologies

This work may not be copied or reproduced in whole or in part for any commercial purpose. Permission to copy in whole or in part without payment of fee is granted for nonprofit educational and research purposes provided that all such whole or partial copies include the following: a notice that such copying is by permission of Mitsubishi Electric Research Laboratories, Inc.; an acknowledgment of the authors and individual contributions to the work; and all applicable portions of the copyright notice. Copying, reproduction, or republishing for any other purpose shall require a license with payment of fee to Mitsubishi Electric Research Laboratories, Inc. All rights reserved. 



\title{
AUTONOMOUS FIELD-DEPLOYABLE DEVICE FOR THE MEASUREMENT OF PHOSPHATE IN NATURAL WATER
}

\author{
Conor Slater ${ }^{\mathrm{a}}$, John Cleary ${ }^{\mathrm{a}}$, Christina M. McGraw ${ }^{\mathrm{a}}$, William S. Yerazunis ${ }^{\mathrm{b}}$, King Tong Lau ${ }^{\mathrm{a}}$, \\ Dermot Diamond*a \\ ${ }^{a}$ National Center for Sensor Research, Dublin City University, Dublin 9, Ireland +35317005000; \\ ${ }^{\mathrm{b}}$ Mitsubishi Electric Research Laboratories,
}

\begin{abstract}
This work describes the ongoing development of an autonomous platform for the measurement of phosphate levels in river water. This device is designed to operate unassisted for one year, taking a measurement every hour and relaying the result to a laptop computer. A first generation prototype has already been developed and successfully field tested. The system contains the sampling, chemical storage, fluid handling, colorimetric data acquisition and waste storage capabilities necessary to perform the phosphate measurement. In addition to this, the device has the embedded control, GSM communications system and power supply to allow independent operation. The entire system is placed inside a compact and rugged enclosure. Further work discussed here builds on the successes of the prototype design to deliver a system capable of one full year of operation. The second generation system has been built from the ground up. Although identical in operation to the prototype its design has a greater emphasis on power efficient components and power management to allow for a longer lifetime. Other improvements include an automated two-point calibration to compensate for drift and a more rugged design to further increase the lifetime of the device.
\end{abstract}

Keywords: Wireless, Sensor, Network, Environment, Phosphate, Microfluidics, LED, Photodiode.

\section{INTRODUCTION}

Phosphate is the most common form of the essential plant and animal nutrient phosphorus. This nutrient is a limiting factor in the growth of most aquatic ecosystems. Eutrophication is the over enrichment of nutrients in natural water. This over abundance of nutrients causes water systems to become overgrown with plants and unsightly algal blooms [1]. These algal blooms are sometimes toxic and so can have an impact on drinking water supplies. This excessive growth is damaging to the aquatic ecosystem as the dissolved oxygen normally available to other animals is depleted which can lead to large scale fish kills $[2,3]$. Human activity is the main source of excessive phosphate and other nutrients. This includes the untreated and treated waste water from sewerage systems, agricultural sources such as fertilizers and animal waste. Other forms are industrial waste products dumped into waterways [4].

Phosphate is normally monitored manually by collecting and filtering samples which are later analyzed in a laboratory. The obvious limitations are the manpower and time cost of this method. The European Water Framework Directive (WFD) requires the monitoring of water systems in the European Community with the goal of maintaining and improving these systems. The manpower required for this task does not exist and so there is an impetus for the development is automated methods of water quality monitoring. Other implications are the limited use "snap-shot" monitoring of a particular waterway's condition will give. Studies have shown the need to understand the spatial and temporal condition of a waterway to asses risk or to classify a particular water system $[6,7,8]$.

An autonomous system for the measurement of nutrients with phosphate in particular is needed. This system will have to operate over extended periods of time without human assistance - ideally 1 year. The system under development described here is based on the yellow vanadomolybdophosphoric method of phosphate detection. This reagent is chosen specifically for its long lifetime [9]. The water sample and the reagent are mixed at a 1:1 ratio. After the reaction takes place the solution will absorb below $400 \mathrm{~nm}$ wave lengths. A prototype of the system has already been built and tested 
[10]. This system contains all the elements needed for a fully autonomous system utilising wet chemistry. The phosphate measurement is based around a microfluidic chip that mixes the reagent and sample. The reagent is stored in a polymer bag and the sample is collected through a filter membrane. The two liquids are pumped through the microfluidic chip where they are mixed using a miniature peristaltic pump. A photodiode and UV LED are used to make and absorbance measurement. Waste from the phosphate measurement is then collected in a second polymer bag. The entire process is conducted by a microcontroller unit which is used for logging the measurement. The system also demonstrates its field deployable requirement: It will most likely be deployed in a remote location so a wireless communication system is necessary. A GSM modem is utilised to relay the data obtained to a laptop computer. Logged data can also be downloaded via a direct serial connection. The sensor is encased in two polycarbonate boxes to protect it from the environment.

Although this system shows that phosphate can be measured automatically using the chosen reagent, power is a major factor in lifetime of this sensor. A single 12V lead acid battery allows for only 7 days of operation taking 2 measurements per hour. This paper describes the design of a new system that incorporates the successful components of the prototype system, but with the new design having a greater emphasis on power efficiency, robustness and overall sensor endurance. Power efficient components are chosen for fluid handling, control and communications. The sensor also has a power management system that allows components that are not in use to be switched off completely, thus not consuming power. The sensor also utilises a two point calibration and cleaning routine to reduce sensor drift.

\section{SENSOR DESIGN}

The layout of the new system is shown in figure 1. The system contains bottles for storing the reagent, calibration solutions and cleaner; A sample port for collecting the water sample to be analysed; An array of solenoid pumps, instead of a peristaltic pump, are used for pumping the required liquids through the microfluidic chip. The microfluidic chip allows for the mixing of the reagent and sample. The chip also presents the reacted sample to a photodiode and LED for an absorbance measurement. The analyzed sample is then pumped to the waste storage. All of the fluid handling and analytical components are controlled by a microcontroller that also performs the data acquisition and stores the data in a flash memory unit. A GSM modem is used to communicate the data via the SMS protocol to a laptop computer. This system differs from the previous in that it has an emphasis on endurance.

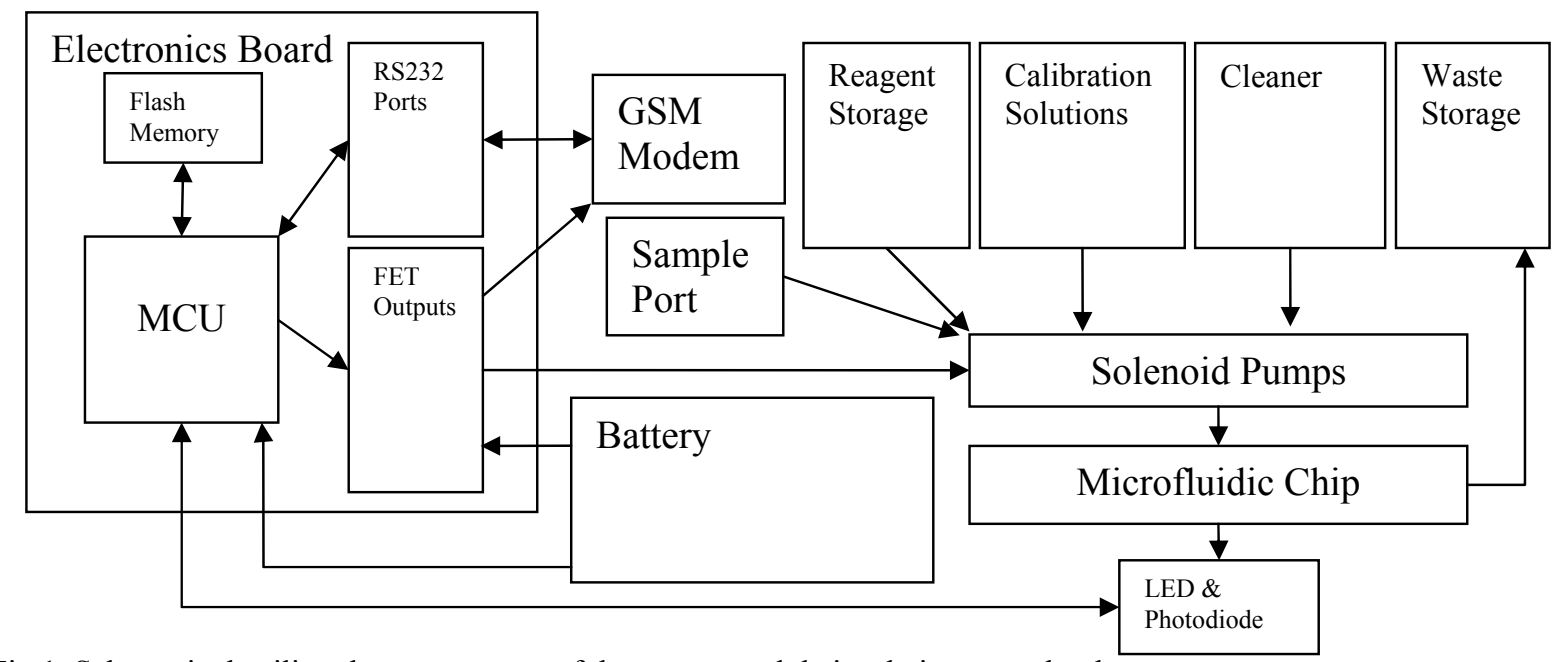

Fig.1. Schematic detailing the components of the system and their relation to each other. 
There are four factors that will determine the sensor lifetime. First is the overall robustness of the sensor. It will have to withstand the environment it is placed. In this sensors case it will have to completely waterproof and be composed of materials that will not corrode in wet conditions. All the components must also operate properly for the duration of the sensors lifetime. The second factor is the amount of reagent and waste storage the sensor in capable of. The amount of reagent and sample that is used for each measurement has to be minimized. The greater the volume of liquid used per measurement limits the amount of measurements that can be taken, limiting the life of the sensor. Larger storage and waste container will increase the size and weight of the sensor and decrease the portability. The third factor is sensor drift. The limit of detection will increase proportionally to the length of time the sensor is operating. This limits the usefulness of the sensor over time. The fourth and most important factor is power consumption. The sensor will only last as long as its battery. More battery capacity will also increase the size and weight of the sensor. This leaves an emphasis on power efficient components and power management. This section describes the reasoning behind the selection and design of the various components of the new system.

\subsection{Chemistry}

The molybdenum blue method is the most popular approach to phosphate measurement. This reagent forms a deep blue complex when added to a sample containing phosphate and absorbs strongly between 650 and 700nm. In this phosphate measurement system in order to reduce the amount of reagent and sample that is used for each measurement a microfluidic chip is used. The blue method is not suitable for use with a microfluidic chip as a precipitate is formed which would block the channels. The major drawback is that the reagent is only stable over a limited period of time. As the sensor lifetime may have to be up to one year, another form of chemistry has to be used. The reagent used is the molybdovanadophosphoric acid method (yellow method) and has been shown to have a lifetime of over one year[9]. This reagent forms no precipitate so can be used in a microfluidic chip. The reagent is mixed at a 1:1 ratio with the sampled water. This simplifies the design of the fluidic chip. The reaction takes place over 2 to 60 minutes depending on temperature and absorbs below 400nm. This allows the use of an LED and Photodiode for the absorbance measurement $[11,12]$. The only major drawback of this reagent is that it is composed of an aggressive acid that readily corrodes stainless steel. The means than all wetted components have to be made for inert plastics.

All solutions were prepared with Milli-Q deionized water (Milli-Ro Plus 30 System, Millipore, Billerica, MA). A 50 mg. $\mathrm{L}^{-1}$ stock solution of phosphorus, in the form of phosphate, was made by dissolving $0.2195 \mathrm{~g}$ of anhydrous potassium dihydrogen phosphate (60218, Fluka, Buchs, Switzerland) in 1 L of deionized water. $2.25 \mathrm{~mL}$ of sulfuric acid (S/9200/PB17, Fisher Chemicals, Leicestershire, England) was added to preserve the stock solution. $0-20 \mathrm{mg} / \mathrm{L}$ standard solutions were prepared from dilutions of the stock solution with deionized water. The reagent was prepared by dissolving $7.143 \mathrm{~g}$ of ammonium molybdate (A-7302, Sigma, Dorset, England) and $0.358 \mathrm{~g}$ of ammonium metavanadate (20555-9, Aldrich, Dorset, England) in $95 \mathrm{~mL}$ of concentrated hydrochloric acid (37\% wt. in water) (926, BDH Laboratory Supplies, Dorset, England) and $905 \mathrm{~mL}$ of water. Deionized water is used as the cleaner for the microfluidic chip between measurements.

\subsection{Microfluidics}

A microfluidic chip is used as a platform for the phosphate measurement. This allows for small volumes of reagent and sample to be used for each measurement $-20 \mu \mathrm{L}$ of both liquids. The reagent and sample are mixed in a T-mixer which leads into a serpentine channel where the resulting solution is presented to the emitter and photo-detector for the absorbance measurement. Each side of the T-mixer has three inlet channels leading into them. On one side are the two phosphate standard solutions for a two point calibration. A third inlet channel is for the water sample. On the other side of the T-mixer there is an inlet for the reagent and two inlets for deionized water for cleaning the microchannels. All channels are of $200 \mu \mathrm{m}$ in width and depth.

The chip is manufactured from a 1mm thick PMMA sheet (ME303011, Goodfellow, Cambridgeshire, England). The microfluidic channels are milled into the sheet using the CAT-3D-M6 micromill (DATRON Electronic, Mühltal, Germany) using a $200 \mu \mathrm{m}$ endmill (00680029, DATRON Electronic, Mühltal, Germany). The $35 \times 20 \mathrm{~mm}$ chip is then cut from the sheet. The channels are sealed by bonding a second $35 \times 20 \mathrm{~mm}$ PMMA chip layer using a pressure sensitive adhesive (ARcare ${ }^{\circledR} 8890$, Adhesives Research, Limerick, Ireland). PEEK tubes are inserted into the inlet and outlet ports and are held in place using epoxy adhesive. Figure 2 shows the completed chip. 


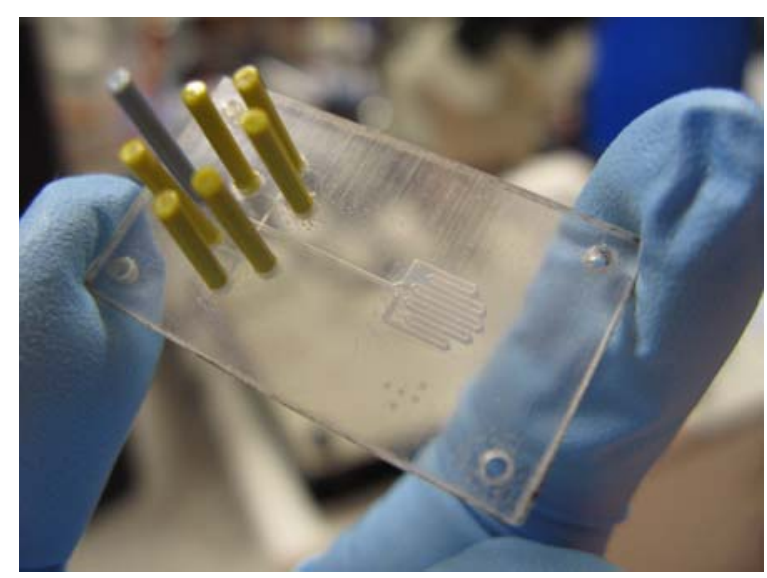

Fig. 2. Finished microfluidic chip. The serpentine channel is visible on the right of the chip and the six inlets and outlet can be seen on the left.

\subsection{Pumps, fluid storage and sample port}

One of the main power consuming components of the previous system is the miniature peristaltic pump (2PP10.S, Eurolink Associates, Tyne\&Wear, UK). This pump is also manufactured from stainless steel so is unsuitable for long term use in the system as it is corroded by the reagent. An array of six miniature solenoid pumps are used in the new system (120SP, Bio-Chem Valve, New Jersey, USA). These pumps are made from PEEK and have Viton seals. The pumps deliver $20 \mu \mathrm{L}$ for each stroke and have $1 / 4$ "-28 UNF ports for tubing interconnects. A $12 \mathrm{~V}$ battery is also suitable for operating these pumps. They are more power efficient than the peristaltic pump. Although they draw $330 \mathrm{~mA}$ of current during operation, their duty cycle is only 1 second per stroke. As opposed to the peristaltic pump that draws $25 \mathrm{~mA}$ over a 2 minute period to pump the same amount of liquid $(20 \mu \mathrm{L})$. In the system there is a pump to draw in the water sample, pump each of the calibration solutions and the reagent. The remaining two pumps are used for the cleaner.

The sample is drawn through a $0.45 \mu \mathrm{m}$ pore diameter filter membrane (Supor ${ }^{\circledR}$, PALL Corporation, Michigan, US) that is held in place between two polycarbonate plates and sealed with O-rings. The port is attached the box and sealed with polyurethane adhesive. The port has a $1 / 4$ "-28 UNF port for connecting tubing.

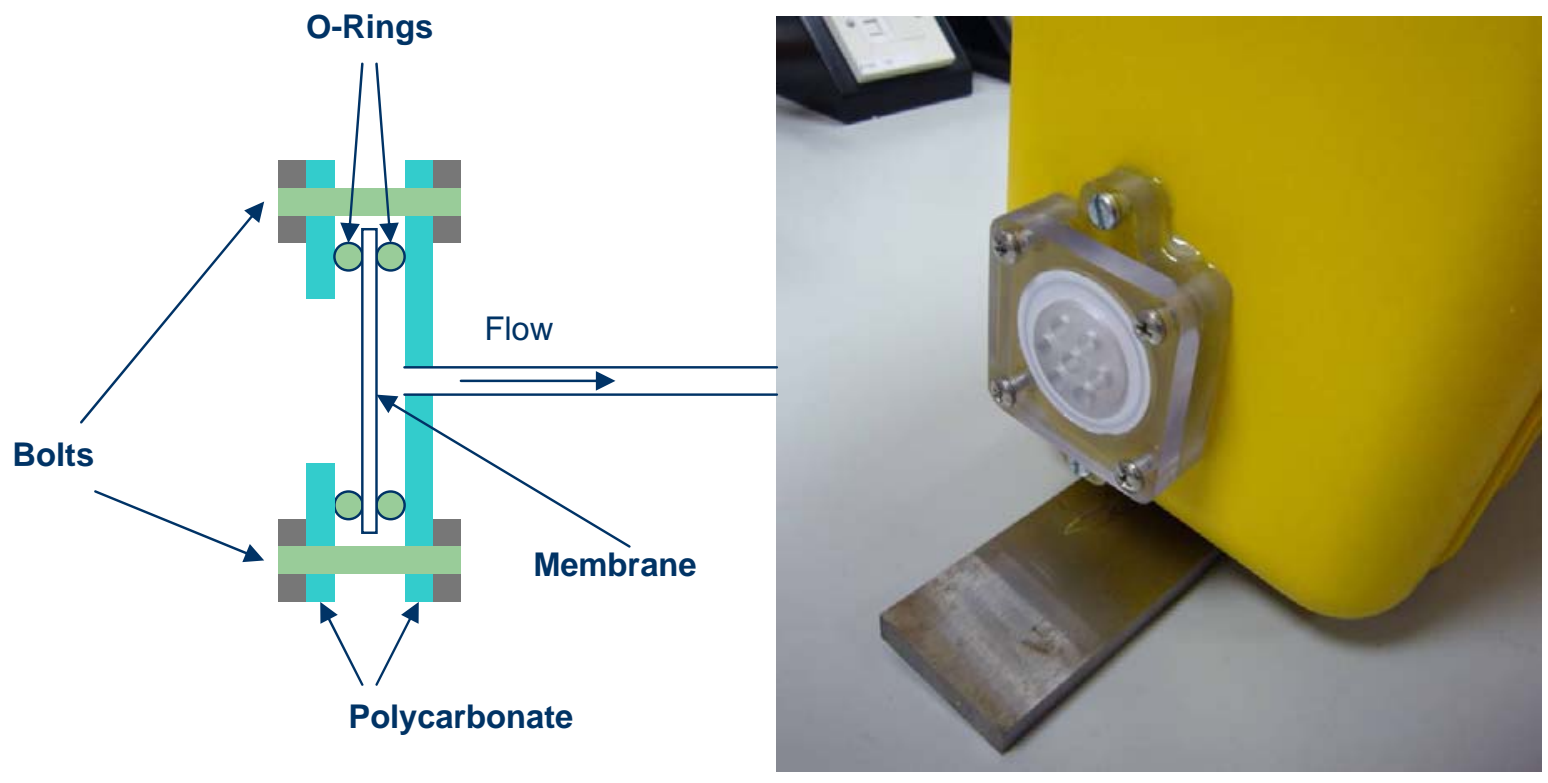

Fig 3. Left: Schematic of sample port. Right: Sample port mounted on phosphate sensor. 
The reagent, calibration solutions and cleaners are stored in five square 250ml HDPE bottles (NALGENE 2018-0250, Nalge Nunc, New York, USA). The reagent storage allows for 12,500 phosphate measurements. Two square 1L HDPE bottles are used for waste storage (NALGENE 2018-1000, Nalge Nunc, New York, USA). The bottle caps (Q-Series, Bio-Chem Valve, New Jersey, USA) used have 1/4" 28 UNF ports and are also fitted with Teflon check valves. The check valve allows air pressure to equalize in the bottle as the liquid is being pumped out. Teflon tubing with $1 / 4$ "-28 UNF connectors (Omni-Lok, Bio-Chem Valve, New Jersey, USA) fitted at the ends, are used to connect the various fluidic components in the system.

\subsection{Data Acquisition, Control and Power Supply.}

The microcontroller used for the control of the system is the MSP430F449 (Texas Instruments). This was chosen for its low power consumption during operational and sleep mode. The MSPGCC compiler also provides an open source platform for software development (mspgcc.sourceforge.net). This microcontroller has, along with numerous digital inputs and outputs, an 8 channel 12bit ADC for data acquisition and two UART ports for communicating with peripheral devices. A 2 megabyte SPI flash chip mounted on the PCB with the microcontroller allows for 16,384 data points to be logged. The solenoid pumps and the power to the GSM modem are controlled via an array of FETs.

A photodiode (S1227-33BR, Hamamatsu Photonics UK Limited, Hertfershire, UK) and a 370nm LED (NSHU550E, Nichia Corporation, Tokushima, Japan) are used for the absorbance measurement. A transimpedance amplifier circuit based around a TLV2772 operational amplifier (Texas Instruments) is used to condition the signal from the photodiode. This circuit is built on a separate PCB from the main controller board. This limits the noise on the signal from the photodiode as it is mounted beside the microfluidic chip. The daughter board is also a mount for a temperature sensor (LM335, ST Microelectronics). The LED is controlled via a BJT and is pulsed during the absorbance measurement. The voltage signals from the transimpedance and the temperature sensor are read by the ADC on the microcontroller.

The entire system is powered by a $12 \mathrm{~V} 7 \mathrm{Ah}$ standard lead acid battery as used in the previous system. The power source is chosen for its low cost, long lifespan and high capacity. The microcontroller monitors and logs the battery voltage with each phosphate measurement.

\subsection{Communication}

A GSM modem is used for communication when the system is deployed. The main reason for the choice of this communications standard is the extensive coverage of the GSM network in Ireland and other countries in the European Union. This means that the phosphate sensor can be deployed to most areas. The modem used is the MC35IT (Siemens, Berlin, Germany) and is also used in the previous system. This particular model communicates via RS232 so allows for easy implementation in the system. In the previous system the GSM modem was always on standby waiting for the user to dial in using a modem to download the data. This method, although simple to implement, is not power efficient. A data call uses as much power as a voice call. Also the modem is always on so it is a constant and unnecessary power drain.

The new system uses the same modem model but uses the GSM modems SMS functionality to send the data to another GSM modem connected to a laptop. This has the advantage of requiring less power as the modem only has to be switched on for 30 seconds to send a message. It can then be switched off completely. This method also has the advantage or requiring less signal strength to operate than a data call. During the operation of the sensor data is collected and then stored in the flash memory. After 24 hours of measurements the all the collected data is sent in bulk via SMS messages.

\subsection{Enclosure}

To withstand the environment that the sensor will be deployed in, it will have to be housed in a rugged enclosure. The enclosure must also radio transparent to allow the modem to communicate and allow for the sample port to be mounted on the outside. A polypropylene case is used for protecting the sensor (1430, Peli Products, Barcelona, Spain). This case is completely water tight and resistant to the reagent used. It is lightweight so does not affect portability. The chemical storage bottles and battery are placed the bottom. While the solenoid pumps and microfluidic chip and LED and photodiode are mounted on a custom ABS mounting plate manufactured using a 3D printer (Dimension SST768, Stratasys, Minnesota, USA). The controller board and GSM Modem are mounted in the enclosure lid. The sample port is mounted on the side of the enclosure. 


\subsection{Assembly}

Figure 4 shows the fully assembled system. The five storage bottles and two waste bottles can be seen at the bottom of the enclosure. The battery sits between the waste bottles. The top plate is flipped up to reveal the six solenoid pumps on the underside. These are mounted with the inlets facing down towards the storage bottles. The outlets can be accessed through the top to allow them to be connected the microfluidic chip. This is housed along with the temperature sensor, LED and photodiode with their accompanying daughter board in the center of the top plate. In the lid of the enclosure the controller board is mounted on the left hand side. The GSM modem along with its antenna is mounted on the right. The sample port not shown in the pictures is mounted on the bottom left hand side of the enclosure.

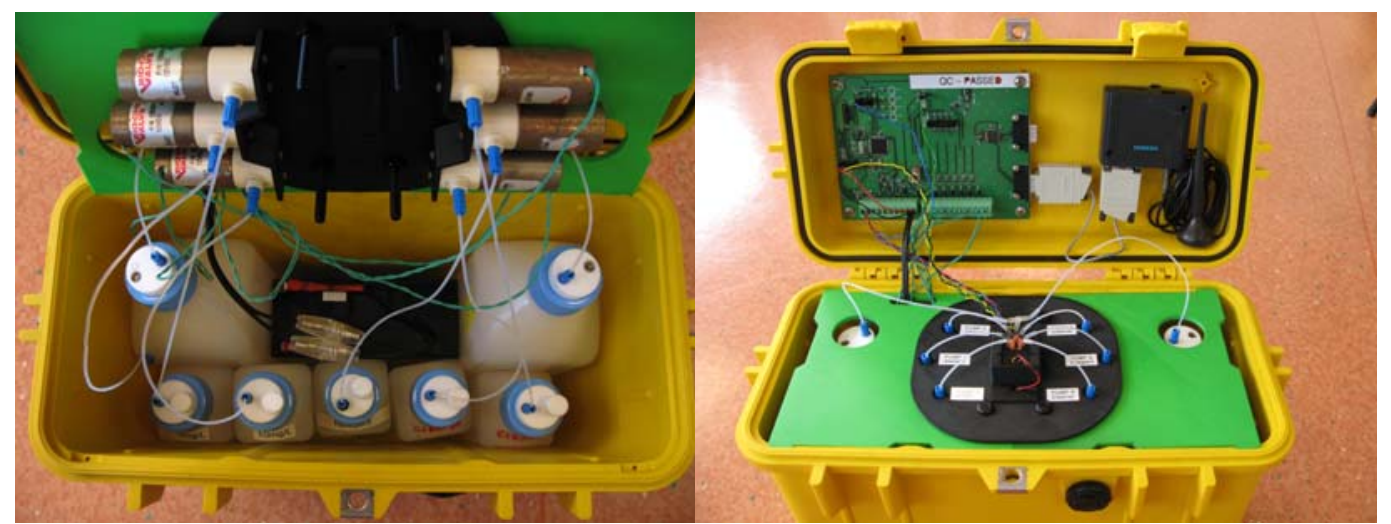

Fig. 4. Fully assembled system. Left: bottom of enclosure showing bottles and battery. Right: Top plate and lid

\section{RESULTS \& DISCUSSION}

To demonstrate the effectiveness of an automated 2 point calibration a 1 day trial was conducted. 0ppm, 10ppm and $50 \mathrm{ppm}$ phosphate solutions where used in the system. A measurement was taken every 10 minutes and an 8 minute period was allowed for the reaction. Between each measurement deionized was pumped through the microfluidic chip to clean it. 144 measurement where taken over the 24 hour period of the experiment. Figure 5 shows the raw data collected. Three traces can be clearly seen, representing the three phosphate standard solutions. The light intensity decreases as the phosphate level increases. Also evident in the plot is a downward drift in all three samples.

In figure 6 it is given that the 0 and 50ppm standard solutions are the known calibration solutions of the phosphate sensor. The $10 \mathrm{ppm}$ phosphate solution is the unknown water sample. According to Beers law the relationship between the absorbance and the quantity of analyte is linear. Using the 0 and 50ppm standard solutions to determine the scale, the level of phosphate in the 10ppm solution can be determined based on the light intensity. This is shown in the plot in figure 6 . The plot of the 10ppm solution shows that the drift is eliminated using this method.

The discrepancy in the first number of measurements is caused by bubbles in the microfluidic chip that form when the sensor is left idle over a period of time. These bubbles are cleared by pumping the various liquids through the chip over the course of the first few measurements.

\section{CONCLUSIONS}

A field deployable sensor for phosphate measurement has been presented. This sensor is an improvement on a previous sensor that demonstrated that a process of phosphate measurement using wet chemistry can be automated in a compact and portable system. The new system uses more power efficient components and power management methods to create a sensor with a longer lifespan. Larger storage containers allow for the sensor to take more measurements. The sensor also has a more rugged design to for it to survive in the environment of its deployment. It has also been shown that an automated 2 point calibration routine can significantly prolong the length of time the sensor is effective at phosphate monitoring. Power is a major issue in the lifetime if the sensor. Future work will involve the implementation of a form of energy harvesting to supplement the battery power supply. 


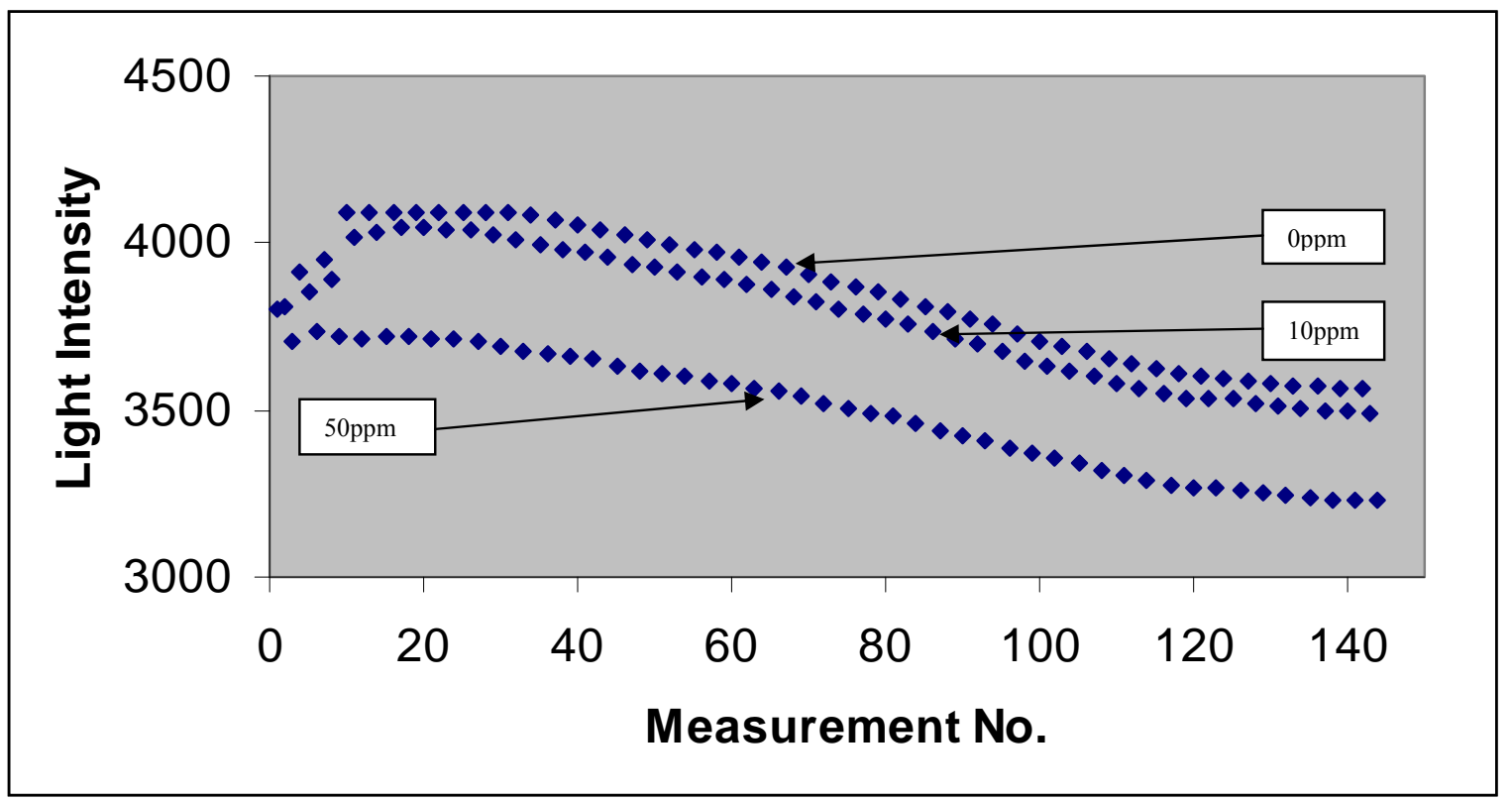

Fig. 5. Raw data from 1 day trial.

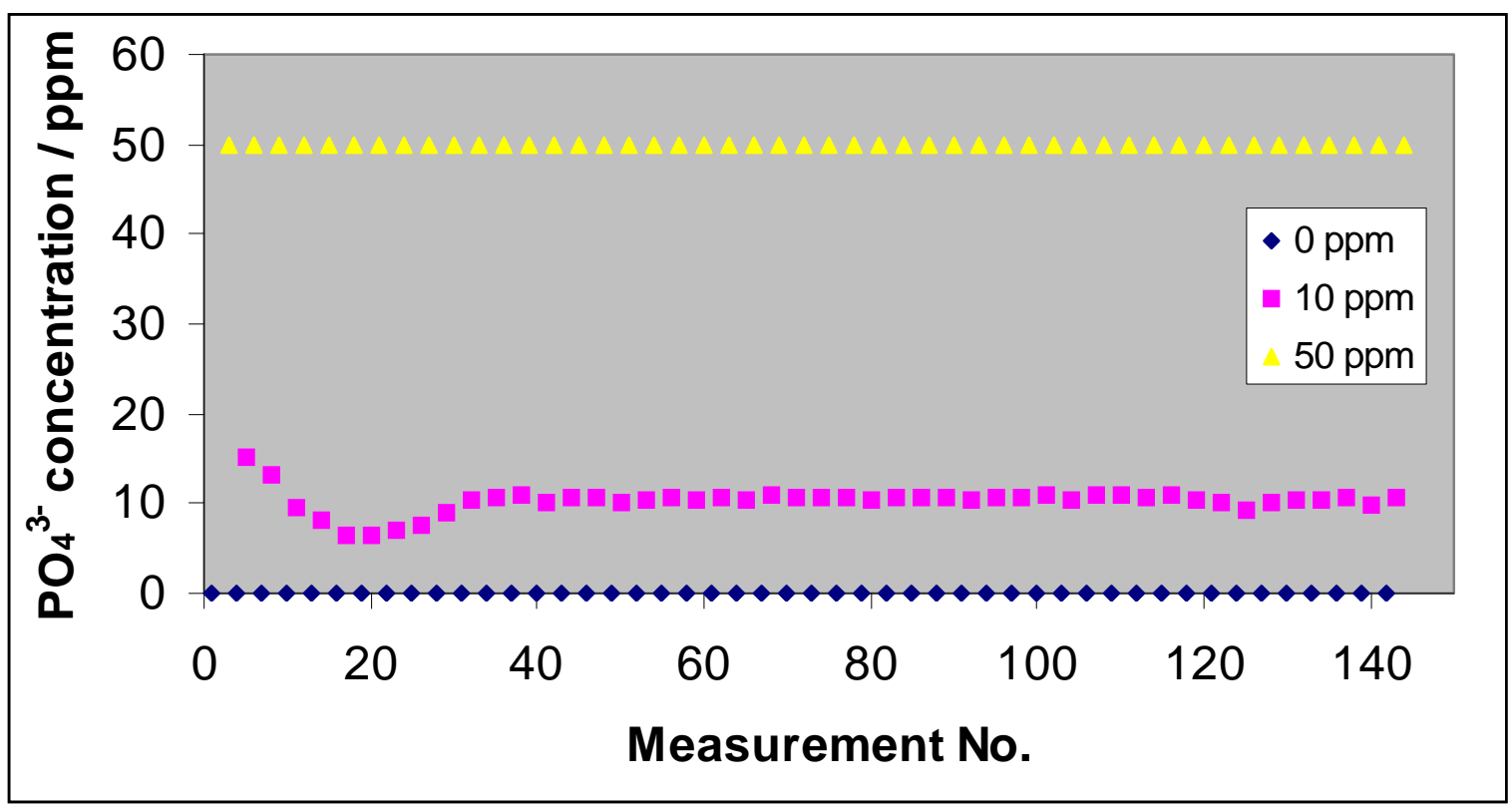

Fig. 6. Drift compensation from 1 day trial.

\section{ACKNOWLEDMENTS}

The Author would like to thank John Barnwell, Jonathan Westhues, Darren Leigh and Paul Dietz for there contribution to this project. This work was carried out under the SmartCoast programme which is funded by the Irish Marine Institute and the Irish Environmental Protection Agency (EPA) under grant AT/04/01/06. The Adaptive Information Cluster is supported by Science Foundation Ireland under grant 03/IN.3/1361. The author would also like to identify MERL as a funding source. 


\section{REFERENCES}

1 K. Robards, I.D. McKelvie, R.L. Benson, P.J. Worsfold, N.J. Blundell, and H. Casey, "Determination of carbon, phosphorus, nitrogen and silicon species in waters," Anal. Chim. Acta, vol. 287, no. 3, pp. 147-299, Mar. 1994.

V.H. Smith, G.D. Tilman, and J.C. Nekola, "Eutrophication: impacts of excess nutrient inputs on freshwater, marine, and terrestrial ecosystems", Environ. Pollut., vol. 100, no. 1-3, pp. 179-196, Aug. 1999.

3 C.P. Mainstone and W. Parr, "Phosphorus in rivers - ecology and management," Sci. Total. Environ. Vol. 282-283, pp. 25-47, Jan. 2002.

D.M. Anderson, P.M. Glibert, and J.M. Burkholder, "Harmful algal blooms and eutrophication: nutrient sources, composition, and consequences," Estuaries, vol. 25, no. 4b, pp. 704-726, Aug 2002.

5 "Directive 200/60/EC of the European Parliament and of the Council of 23 October 2000 establishing a framework for Community action in the field of water policy". Official Journal L 327/1, Dec. 2000.

6 I. Donohue, D. Styles, C. Coxon, and K. Irvine, "Importance of spatial and temporal patterns for assessment of risk to diffuse nutrient emissions to surface waters," J. Hydrol., vol. 304, no. 1-4, pp. 183-192, Aug. 2005.

7 Irvine, K., "Classifying ecological status under the European Water Framework Directive: the need for monitoring to account for natural variability. Aquat. Conserv.: Mar. Freshwat. Ecosyst.," vol.14, no. 2, pp. 107-112, Feb 2004.

$8 \quad$ P. Jordan, J. Arnscheidt, H. McGrogan, and S. McCormick, "High-resolution phosphorus transfers at the catchment scale: the hidden importance of non-storm transfers," Hydrol. Earth Syst. Sci., vol. 9, no. 6, pp 685-691, Dec. 2005.

$9 \quad$ M. Bowden and D. Diamond, "The determination of phosphorus in a microfluidic manifold demonstrating long-term reagent lifetime and chemical stability utilising a colorimetric method," Sens. Actuators B, vol. 90, no. 1-3, pp. 170-174, April 2003.

10 C.M. McGraw, S.E. Stitzel, J. Cleary, C. Slater, D. Diamond, “Autonomous microfluidic system for phosphate detection," Talanta, In press, available online at http://www.elsevier.com/locate/talanta

11 M. Bowden, M. Sequiera, J.P. Krog, P. Gravesen, and D. Diamond, "Analysis of river water samples utilising a prototype industrial sensing system for phosphorus based on micro-system technology," J. Environ. Monit. Vol. 4, no. 5, pp. 767-771, Aug. 2002.

12 M. Bowden, M. Sequiera, J.P. Krog, P. Gravesen, and D. Diamond “A prototype industrial sensing system for phosphorus based on micro system technology,” Analyst, vol. 127, no. 1, pp. 1-4, Jan. 2002.

13 M. Bowden, O. Geschke, J.P. Kutter, and Dermot Diamond, "CO2 laser microfabrication of an integrated polymer microfluidic manifold for the determination of phosphorus,” Lab Chip, vol. 3, no. 4, pp. 221-223, Sept. 2003. 